\title{
REVISITANT LES FACTEURS AFFECTANT L'UTILISATION DES TICS SUR L'INFORMATION RELATIVE AUX INTRANTS AGRICOLES PAR LES PAYSANS DANS LES PAYS EN VOIE DE DEVELOPPEMENT D'AFRIQUE ET ASIE
}

\section{Macire Kante}

School of Computing and Informatics, University of Nairobi, Nairobi, Kenya

Agence des Technologies de l'Information et de la Communication, Bamako, Mali

Christopher Chepken

School of Computing and Informatics, University of Nairobi, Nairobi, Kenya

Robert Oboko

School of Computing and Informatics, University of Nairobi, Nairobi, Kenya

\section{RESUMÉ}

Malgré une exposition des paysans aux Tics sur l'information de production agricole, la contribution de ceux-ci dans ce domaine n'est encore satisfaisante. Par conséquent, une étude a été menée pour informer notre audience sur les facteurs affectant l'utilisation de ces Tics par une révision des Tics et les articles publiés sur ces Tics en utilisant la Théorie Ancrée. Les facteurs avantage relatif, compatibilité, simplicité, l'influence sociale, observabilité, alphabétisation, savoir-faire Tics et qualité de l'information affectent positivement l'utilisation de ces Tics tandis que et le cout excessif des Tics affectent négativement leurs utilisations.

Mots-clés: Tics, agriculture, intrants agricoles, paysans, accès, utilisation

\begin{abstract}
Despite farmers' exposition to ICTs on agricultural input information, their contribution to the access and use of agricultural input information is far from expectation. This study therefore, reviewed these ICTs in developing countries to extract the factors that affect ICTs' adoption by small-scale famers using the Grounded Theory. The factors Relative Advantage, Compatibility, Simplicity, Social Influence, Observability, Literacy, ICTs' Skills were identified as affecting positively ICTs' use while the High Cost was identified as negatively affecting these ICTs.
\end{abstract}

Keywords: ICTs, Agriculture, farm inputs, farmers, access, use

Manuscript first received/Recebido em: 2017/May/25 Manuscript accepted/Aprovado em: 2017/Aug/09

Address for correspondence / Endereço para correspondência:

Macire Kante, School of Computing and Informatics, University of Nairobi, Mali. E-mail: maciredancira@gmail.com http://orcid.org/0000-0001-5425-4549, https://macirekante.wordpress.com

Christopher Chepken, School of Computing and Informatics, University of Nairobi, Kenya. E-mail: chepken@uonbi.ac.ke

Robert Oboko, School of Computing and Informatics, University of Nairobi, Kenya. E-mail: roboko@uonbi.ac.ke

Published by/ Publicado por: TECSI FEA USP - 2017 All rights reserved. 


\section{INTRODUCTION}

L'agriculture constitue le pilier de l'économie des pays en voie de développement. Par exemple, Bwalya (2009) affirme que l'agriculture constitue le pivot de l'économie Ethiopienne. Au Mali, elle contribue 37\% au Produit Intérieur Brut (PIB) du pays en 2008 (Angelucci, Gourichon, Mas Aparisi, \& Witwer, 2013). En Tanzanie, Siyao (2012) rapporte que le secteur agricole constitue le maillon essential de l'économie Tanzanienne.

Les petits producteurs dominant cette agriculture. Par exemple, les petits producteurs dominant les activités agricoles en Tanzanie, au Mali et en Ethiopie (Angelucci et al., 2013; Bwalya, 2009; Siyao, 2012). Ceci est semblable au cas de plusieurs pays en voie de développement.

Néanmoins, des études ont démontré que ces activités agricoles sont marqués par un rendement faible à l'hectare pour les cultures vivrières et rentes (AGRA, 2014; Murage, Midega, Pittchar, \& Khan, 2013; Traoré, Mamy, JBélières, \& Hilhorst, 2011). L'utilisation des intrants agricoles (engrais, conseils agricole, climat...) est essential pour augmenter le rendement de ces principales cultures. The International Development Fertilizer Centre (2004) conclut qu'une intensification durable des activités agricoles ne peut être atteinte sans utilisation d'intrants agricoles appropriés. De façon générale, les paysans devraient améliorer leur rendement agricole à travers l'accès aux intrants agricoles et utilisation de ces intrants.

Toutefois, l'accès et l'utilisation de ces engrais dépend en grande partie de la disponibilité l'information. Par exemple en Tanzanie, la décision des paysans d'adopter (utiliser) des engrais agricoles est fortement influence par la disponibilité d'information (Msoffe \& Ngulube, 2016). Par conséquent, les technologies de l'information et de la Communication ont un important rôle à jouer dans la diffusion de ces informations pour permettre aux paysans d'accéder aux intrants agricoles et de les utiliser afin d'augmenter leur rendement et par la leur production agricole.

Les Technologies de l'Information et de la Communication (Tics) $^{1}$ jouent un rôle crucial dans la diffusion de l'information agricole sur les intrants agricoles au niveau des petits producteurs des pays en voie de développement d'Afrique et d'Asie. Une revue empirique du travail scientifique abattu durant les quinze dernières années (Aker, 2011; Chung, 2015; Kameswari, Kishore, \& Gupta, 2011; KTM, 2013; Ouedrago, 2008; T. Palmer, 2014; Wulystan \& Andrew, 2013) a fourni une idée générale de l'utilisation des Tics par les paysans de l'accès et l'utilisation de l'information sur les intrants agricoles. Pourtant, la cause de l'insignifiante contribution des Tics dans l'accès et l'utilisation de l'information sur les intrants agricoles n'est pas claire.

Alors qu'il a été bien établi que les paysans ont été exposé à ces Tics pour plus d'accès et d'utilisation de l'information agricole sur les intrants (Bosch et al., 2012; Kaddu, 2011; Kameswari et al., 2011; Mittal \& Mehar, 2012). Il n'y'a pas de compréhension claire si les

\footnotetext{
${ }^{1}$ Par Tics, nous pardons des nouvelles Tics tels que le téléphone portable et les telecentres.
} 
paysans ont accès à l'information agricole sur les intrants et s'ils utilisent ces informations. Par exemple en Inde, l'utilisation de IFFCO-Airtel Kisan (Indian Farmers Fertilizer Cooperative Limited and Airtel - un service Tic pour les paysans indiens relative à l'information agricole sur les intrants lances en 2008) par les paysans est très faible (Kameswari et al., 2011). Une importante question à répondre est alors : quels sont les facteurs affectant l'utilisation des Tics par les paysans pour accéder et utiliser l'information agricole sur les intrants et comment ces facteurs affectent ils l'utilisation de ces Tics?

Cette question a été en partie répondue dans une publications (Kante, Oboko, \& Chepken, 2016). Néanmoins, elle reste d'actualité et d'autres facteurs pourraient être identifiés.

Le document est ainsi organisé : la section méthodologie de la recherche répond à la question comment avons-nous conduit cette recherche ; la section suivante résultats présente les résultats et la section discussion les compare avec d'autres travaux en lien avec le nôtre et enfin la section conclusion présente ce qu'implique nos résultats pour le monde scientifique spécialement le monde des Tics et fourni les limitations de l'étude et recommandations pour des futures études.

\section{METHODOLOGIE DE LA RECHERCHE}

\subsection{Sources de Données}

Premièrement, nous avons revisité quelques Tics (sélectionnés) relatives aux informations agricoles sur les intrants en Inde, Indonésie, Sénégal, Tanzanie, Kenya, Pakistan, Chine et Mali.

Deuxièmement, afin de mener efficacement notre recherche systématique selon la théorie ancrée (Wolfswinkel, Furtmueller, \& Wilderom, 2011), nous avons définis des critères d'inclusion ou d'exclusion pour qu'un article soit inclus dans notre ensemble de données. Le principal critère était que l'article devrait avoir été publié entre 2001 et 2017. Si tel n'était pas le cas, l'article était exclu de notre ensemble de données. Ensuite, les champs de recherche ont été définis et les termes spécifiques à utiliser dans la recherche ont aussi été définis (Table 1). 
Table 1. Source approprie de recherche et les termes de recherche

\section{Inclusion}
Temps
Entre 2001-2017

Champs de recherche

Source appropriée de recherche
Système d'Information; Informatique; Agriculture

Researchgate.net, Google scholar, sciencedirect.com, aisnet.org, PubMed, webofscience.com
Termes de recherche
Tics; ICT4D; Agriculture; intrants agricoles; pays en voie de développement; information; accès à l'information agricole sur les intrants; utilisation de l'information agricole sur les intrants; Tics dans les pays en voie de développement; Tics et agriculture; Tics dans l'agriculture; Tics sur l'information agricole sur les intrants; Contribution des Tics dans le domaine agricole; Céréales.

Après une longue période de recherche, nous peaufinions notre échantillon selon les critères définis dans la Table 1. Notre premier ensemble de données était constitué de 53 études sur les Tics dans le domaine de l'information agricole sur les intrants publiés entre 2001 et 2017 dans : MIS Quarterly, Computers and electronics in agriculture, Journal of Research in International Business and Management, International Food Policy Research Institute, Journal of Chemical Information and Modelling, ICT in Agriculture Sourcebook, Electronic Journal of Information Systems in Developing Country, International Food Policy Research Institute, Computers \& Education, Agricultural Economics, The information manager, Journal of Agricultural Extension, American Journal of Economics and Business Administration, GSMA, Journal of Agriculture and Social Research (JASR), Interactive Educational Multimedia, American Journal of Health Behaviour, Journal of Research in International Business and Management and Economics of Agriculture.

Finalement, nous consignons ces articles avec les services Tics que nous avions révisés dans les pays en voie de développement pour faire ressortir les facteurs affectant ces Tics.

\subsection{Description des Données}

De notre ensemble de données, nous avons extrait : 1) Information sur la disponibilité des Tics dans le domaine de l'information agricole sur les intrants dans les pays en voie de développement ; 2) Facteurs affectant l'utilisation de ces Tics par les paysans dans les pays en voie de développement dans l'accès à l'information agricole sur les intrants (agricoles) et l'utilisation de cette information.

\subsection{Analyse des Données}

Nous avons applique les principes de la Théorie Ancrée (ou la Théorisation Ancrée) (Urquhart \& Fernández, 2013). La théorie ancrée est une méthode de recherche venant des 
sociologues Barney G. Glaser et Anselm L. Strauss's $(1965,1967)$ qui vise à construire des théories non pas à partir d'hypothèses mais à partir des données (Charmaz, 2006). En lieu et place d'hypothèses, le chercheur recueille des données et à partir de ces données, construit son modèle (ou sa théorie) (Urquhart \& Fernández, 2013). Cette technique est bien recommandée dans le cadre d'une revue littéraire et surtout dans le domaine des systèmes d'information (ibid.). Par conséquent, la méthode est propice à notre cas, ou nous cherchons à construire un modèle conceptuel à partir des facteurs empiriques pour l'utilisation des Tics pour l'accès à l'information agricole sur les intrants et l'utilisation de cette information par les paysans dans les pays en voie de développement.

Les principes de la Théorie sont: codage libre; codage axial et le codage sélectif (Urquhart \& Fernández, 2013).

Nous avons choisi, lu et souligné tout résultat que nous trouvions en lien avec notre sujet et les questions de recherche dans chacun des articles. Chaque mot, phrase ou paragraphe que nous avions souligné (met en relief) dans chacun des articles représentait un extrait relevant de notre étude. Nous recommençons à relire encore ces extraits. Durant cette procédure de relecture, un certain nombre de concepts qui capturaient le sens ou une partie du sens de ces extraits ainsi que leurs articles correspondant ont été ré-analysés et synthétisés. Ceci nous conduit à incorporer chacun de ces extraits dans un concept. Ce travail d'extraction et de regroupage sous forme de concept était le principe de codage libre de la Théorie Ancrée selon Wolfswinkel et al. (2011). Après cela, nous procédions avec le codage axial qui consistait à regrouper les concepts identifies en catégories. Ceci nous conduit par la suite à intégrer les différentes catégories de manière à répondre à nos questions de recherche. Cette procédure est connue sous le nom de codage sélectif de la Théorie Ancrée.

\section{RESULTATS}

Cette section présente les résultats de notre étude.

\subsection{Quelques Tics sur l'information agricole sur les intrants dans quelques pays en voie de développement}

Dans cette partie, nous fournissons une brève description des services Tics de quelques pays en voie de développement. L'objectif est de démontrer qu'ils opèrent dans le domaine de l'information sur la production agricole.

\subsubsection{Expériences venant de l'Inde}

Les Technologies de l'Information et de la Communication sont supposées jouer un rôle central dans l'amélioration des opérations des producteurs en facilitant l'accès des intrants agricoles à travers une meilleure information sur ces derniers pour un meilleur rendement agricole. Dans une étude nationale sur les paysans indiens, il avait été retrouver que les petits producteurs avaient en effet bénéficié d'une augmentation de commodité (dans la recherche de l'information de production agricole) et une baisse des coûts en utilisant leurs téléphones portables dans la recherche d'information agricole tel que la disponibilité des intrants agricoles (Mittal \& Mehar, 2012). Certains services (Tics) ont été implémentés en Inde pour fournir de l'information agricole sur les intrants aux petits producteurs. 


\section{E-choupal}

E-choupal est un service Tic Indien visant à construire un lien entre l'agriculteur et l'agro-industriel. Le model a été développé dans le souci de s'attaquer aux problèmes des agriculteurs fragmentés, une infrastructure faible, un nombre élevé d'intermédiaires dans le secteur agricole Indien (Sukhpal, 2004). Le service diffuse :

i) De l'information en temps réel et personnalisée aux agriculteurs afin d'améliorer leur capacité à prendre des décisions pour un meilleur alignement de leur production avec le marché et aussi améliorer la qualité de leur produits agricoles.

ii) De l'information sur l'accès ainsi que les prix des intrants agricoles sont délivrés au niveau du village aux producteurs.

Pratiquement, les agriculteurs peuvent accéder aux toutes dernières informations sur la pluviométrie, aux meilleures techniques agricoles en fonction du type de sols, les prix des intrants agricoles sur le marché dans le village à travers un portail. E-choupal facilite aussi le ravitaillement des agriculteurs en intrants agricole dans le village.

Le besoin en information agricole sur les intrants des agriculteurs traité par e-choupal sont : information pluviométrique (climat), conseils sur le cycle agricole, meilleurs technique de semis par type de semence et par type de sol, prix des intrants agricoles (semences, engrais,...) ainsi que des pesticides (Choupal, 2011; Dangi \& Singh, 2010).

\section{IFFCO Airtel initiative}

Cooperative des Agriculteurs Indiens (Indian Farmers Fertiliser Cooperative Limited IFFCO) et Airtel (opérateur de téléphonie mobile) ont lancé en 2008 un service (TIC) pour fournir de l'information agricole sur les intrants en direction des agriculteurs indiens (Singh, Bhanotra, L, Wani, \& Kumar, 2016). Les agriculteurs peuvent acheter un téléphone portable déjà enregistre pour le service et chercher de l'information agricole sur les intrants à travers des SMS (Short Message Service) ou un centre d'appel téléphonique.

\subsubsection{Expériences Indonésiennes}

\section{Nokia Life}

Nokia Life suite est un service d'information à travers un téléphone portable spécifique (Nokia) lancé en Inde en 2009, agrandi la même année à l'Indonésie et actuellement même au Nigeria et en Chine. Le service est moins cher en Indonésie que dans les quatre autres pays. Son cout est: 5 Yuan Chinois (0.76US\$) en Chine par mois, 60 Rupée Indien (0.88US\$) en Inde par mois, 500 Rupiah Indonésien (0.03US\$) en Indonésie par mois et 250 Naira Nigérian (1.25US\$) au Nigeria par mois (Pshenichnaya \& Clause, 2013). Le service a un leadership sur le marché Indonésien (Ueno \& Yoshida, 2012). L'Indonésie a été choisie dans cette étude parce que l'expérience Indonésienne est différente des trois autres pays. Nokia Life délivre des services dans le domaine de l'éducation, la santé, l'agriculture ainsi que des documentaires a but distractif afin de résoudre le manque d'information et permettre ainsi aux consommateurs dans les sociétés émergentes d'être bien informé et améliorer leurs vie (Pshenichnaya \& Clause, 2013). Le service délivre de l'information agricole sur les intrants à travers des SMS. Nokia 
Life Agriculture services fournis aux agriculteurs les types de semences par type de sols, le prix des produits agricoles sur le marché, l'information pluviométrique (climat) et des conseils agronomiques en utilisant des SMS.

\subsubsection{Expériences Tanzanienne}

\section{TigoKilimo}

TigoKilimo est un service agricole a valeur ajoutée (Agricultural Value Added Service VAS), mis en marche par l'opérateur de téléphonie mobile Tigo en Tanzanie. Le service offre aux paysans à travers le téléphone portable des informations pertinentes, a temps et recevables dans les domaines suivants : agronomie, semences, prix des produits agricoles sur le marché et l'information climat (pluviométrique) Le contenu est accessible à travers trois moyens : Unstructured Supplementary Service Data (USSD), Voix réponse interactive (Interactive Voice Response -IVR) et une ligne d'aide (Chung, 2015). Les utilisateurs de TigoKilimo, affirment que le service leur ai bénéfique: meilleur rendement agricole confirme par $(45 \%$ des répondants), meilleure connaissance des pratiques d'ensemencement $(37 \%)$ et une meilleure habilité à prédire le climat pour le début de la campagne agricole (23\% des répondants) (Chung, 2015).

Certains agriculteurs ont témoigné à propos de ce service TIC. Par exemple, "Auparavant je désherbais mon champs, enlevait toutes les mauvaises herbes de mon champs et les jeter hors du champ. Mais avec TigoKilimo, j'ai su que je ne devrais pas jeter ces mauvaises herbes hors du champ, mais plutôt les laisser pourrir sur place dans mon champ. Ainsi, je fais maintenant comme ils me conseillent" (Chung, 2015). Dans le même secteur, Zantel (opérateur de téléphonie mobile) lança aussi Z-Kilimo.

\section{Z-Kilimo}

Z-Kilimo est un service à base d'SMS en Tanzanie, utilisant le téléphone portable pour fournir aux agriculteurs l'accès à des méthodes de culture compréhensible. Les paysans peuvent accéder au service en tapant sur leur téléphone *149*50\#. Un menu s'affichera alors avec des informations sur le climat (pluviométrie), des détails sur les sols, des méthodes de désinsectisation et des informations sur l'élevage (ZANTEL, 2013).

\subsubsection{Expériences Kenyannes}

Le Kenya a fait des progrès significatifs dans l'accroissement du rendement agricole grâce à un accès accru des paysans aux crédits, aux information agricoles, système d'information agricole, une solide relation entre les agriculteurs et les réseaux de distributions des produits agricoles et l'utilisation des intrants agricoles (KTM, 2013).

\section{M-farm}

M-Farm est un service de téléphone portable et une plateforme web avec comme objectif d'améliorer le secteur agricole Kenyan à travers la connexion des paysans entre eux d'abord parce que la collaboration entre paysans participe d'une meilleure diffusion de l'information agricole (World Bank, 2011). Le service assiste les paysans à savoir quand cultiver et les connecte aux acheteurs (MFARMERKENYA, n.d.). Le paysan peut avoir le prix des intrants 
agricoles et prendre une décision à quand payer ces intrants et ou les payer. Avec M-Farm, les paysans Kenyans peuvent envoyer des messages à partir de leurs téléphone portable pour avoir des informations de vente de leurs produits agricoles, acheter directement les intrants agricoles avec les fabricants à un prix abordable et aussi trouver des acheteurs de leurs produits agricoles (Odhiambo, 2014).

NAFIS

Le Service National d'Information des Paysans (National Farmers Information Service NAFIS) est un service agricole à valeur ajoutée. Il intègre une application mobile et un service internet fournissant de l'information agricole aux paysans. Les agriculteurs peuvent accéder à ces informations soit en envoyant des SMS ou en appelant directement (Odhiambo, 2014).

\section{MFarmer}

MFarmer Kenya est une branche de Zevan Limited qui est concentré dans les pays en voie de développement utilisant le téléphone portable pour diffuser de l'information agricole.

Ces trois applications (Mfarm, $\mathrm{mFarmer}$ et Nafis) ont déverrouillé la porte pour les petits producteurs pour une meilleure commercialisation de leurs produits agricoles et un meilleur accès aux informations dans les cinq régions de Nairobi, Kajiado, Narok, Nyandarua et Nandi (Odhiambo, 2014).

\subsubsection{Expériences Pakistanaises}

\section{Ukisaan}

Ukisaan est service agricole a valeur ajoute lance par Ufone (un opérateur de téléphonie) pour les paysans pakistanais. Avec Ukisaan les paysans écoutent les informations relatives aux semences agricoles et des informations sur la pluviométrie (climat) dans leur langue locale. Il fournit aussi des informations agricoles à travers un centre d'appel téléphonique. UKissan est disponible seulement pour les clients de l'opérateur de téléphonie.

Kissan

Celui-ci est un service agricole à valeur ajoutée géré par ZONG (un opérateur téléphonie) au Pakistan pour ces clients. Il fournit des informations agricoles sur les intrants à travers un portail (engrais, pesticides, pluviométrie et conseils agronomiques)

\subsubsection{Expériences Chinoises}

\section{NOVA}

La diffusion de l'information agricole est assurée par NOVA (système d'information agricole) (CICC, 2003). C'est un service diffusant de l'information agricole à travers le web. Il y'a des utilisateurs du système qui ont fait des rendements agricoles remarquable en utilisant certaines fonctions du service tels que l'achat des intrants agricoles. D'autres utilisateurs suggèrent même que sans le service, ils ne peuvent pas mener d'activités agricoles. Nova est généralement appréciée par les agriculteurs, démontrant qu'il apporte l'assistance nécessaire. 
En particulier, grâce au système des centres d'appels (que Nova a implémenté, dans un environnement ou l'accès aux ordinateurs sans internet était limité), Nova a pu produire des résultats remarquable pour les paysans chinois (CICC, 2003).

\section{SOUNOUNG}

La diffusion de l'information agricole en Chine est assurée aussi par SOUNOUNG. Ce projet, à travers internet, collecte un ensemble d'information agricoles et les diffuse à destination des paysans (Harrod \& Jamsen, 2010). En 2009, il y avait 1276 ménages qui utilisaient le site web de Sounoung et ces chiffres ont doublé en 2010 (Harrod \& Jamsen, 2010), indiquant par là un succès indisputable du projet.

D'où vient ce succès? Le projet travaille avec les organisations (coopératives) paysannes comme partenaires. Ces coopératives sont bien structurées et fonctionnelles. Ainsi, les membres de la coopérative pouvaient avoir accès aux informations agricoles de SOUNOUNG à travers leurs ordinateurs connectes à internet, téléphone portable (smartphone). En fonction du réseau de connexion, les paysans avaient accès à l'information agricole selon leurs régions et type de sols à cultiver. Pour les paysans qui n'ont pas le savoir-faire d'aller sur internet ou d'utiliser un smartphone, la coopérative pouvait chercher les informations dont ces paysans avaient besoin et les leurs donner sous forme imprimée (Harrod \& Jamsen, 2010).

Dans beaucoup de pays en voie de développement, le téléphone portable est le plus populaire des outils Tics pour accéder à l'information agricole (sur les intrants). En Chine, ceci a été confirmé par une enquête nationale à cause de l'opportunité en termes de temps du téléphone mais aussi de la commodité du téléphone portable (ibid.).

\subsubsection{Expériences Maliennes}

\section{SENEKELA}

SENEKELA (paysan) est un service TIC lance au Mali en 2013 par Orange (Operateur télécom) pour aider les paysans Maliens à augmenter leurs rendement agricole à travers une offre d'information sur les intrants agricoles pouvant les aider dans ce sens (Orange, 2014). Il offre un service d'assistance téléphonique avec un personnel spécialisé dans le domaine agricole qui couvre toute une gamme de sujets et de prix de marché agricoles (T. Palmer, 2014). Le service est actuellement déployé dans deux régions sur les huit que compte le pays.

SENEKELA opère grâce à un centre d'appel téléphonique avec des agronomes qui donnent des conseils (en Français et en Bambara) sur les questions quotidiennes des paysans dans le domaine agricole incluant :: méthodes de culture, semences, temps d'ensemencement et l'utilisation des engrais (GSMA, 2015). L'opérateur télécom (Orange Mali) qui a lancé le projet travaille avec l'Etat à travers l'Institut d'Economie Rurale (IER) et quelques ONG.

\section{MyAgro (Ngasene)}

MyAgro (Ngasene) est une entreprise sociale aidant les petits exploitants ruraux au Mali à casser le cycle de pauvreté en les assistant à prévoir, épargner pour acheter des intrants agricoles pour rendre les champs plus profitables (Myagro, n.d.). Il permet aux paysans d'acheter des intrants agricoles de haute qualité (semences certifies et engrais) sur un système layway grâce 
à une plateforme d'SMS et un réseau de vendeurs locaux. MyAgro aide les paysans à avoir de l'information agricole sur les intrants qui les aidera à augmenter le rendement de leurs cultures en utilisant des méthodes modernes de culture et en fournissant un accès simple aux machines agricoles qui peuvent rendre leur travail plus effectif et profitable. Le service fournit aussi des formations sur le type de sols aux paysans et un accès au marché pour écouler leurs produits agricoles (le surplus agricole). Le model tourne autour de : une plateforme d'SMS et un réseau d'associes locaux dans les villages respectifs qui peuvent travailler avec environ un millier de paysans. Le projet est actuellement concentre sur les céréales notamment le maïs, le sorgho et l'arachide (Myagro, n.d.). Myagro (Ngasene) a démontré un certain succès comme noté ici :

Deux ans auparavant, Amadou visitait le magasin de Myagro durant une foire au cours de laquelle, il rencontrait l'un de nos agents. Ce dernier lui expliqua comment il pouvait considérablement augmenter son rendement. Amadou payait alors un ensemble semencelengrais avec nous utilisant notre système d'épargne layway à travers son téléphone portable. Une année plus tard, Myagro lui délivrait son ensemble et il cultiva (avec sa famille) deux ares et demi en utilisant les semences/engrais myagro. L'année d'avant, avec le même champs, il rapportait seulement 11 sacs de mais, l'année à laquelle il a démarré avec Myagro, il a augmenté son rendement de 40\%. (Myagro, n.d.).

\subsection{Facteurs affectant l'utilisation des Tics relative à l'information agricole sur les intrants}

Cette section présente les résultats selon la méthode de la Théorie Ancrée (Grounded Theorie). Les principaux concepts ont été dégagés dans le codage libre. Ces concepts furent catégorisés dans le codage axial et finalement les propriétés de ces catégories déterminés (codage sélectif). A l'aide des articles de notre ensemble de données, nous avons fait ressortir les facteurs affectant l'utilisation des Tics.

\subsubsection{Codage libre}

Quelques concepts ont été extraits de différentes études.

\subsubsection{Avantage Relatif}

L'Avantage Relatif (ou supériorité) est le degré avec lequel une innovation (Tic), est perçue comme étant meilleure que l'idée de la précédente innovation et plutôt exprimée en terme de rentabilité économique (ici en terme d'accès à l'information agricole sur les intrants et par là, en terme de rendement) et/ou prestige social (Carter \& Belanger, 2004). Ce concept a été observé dans les expériences venant de plusieurs pays d'études. Le Tableau 2 pour plus de détails sur ces pays et articles. Partant de ce fait, nous concluons que l'Avantage Relatif est un facteur dans l'utilisation des Tics.

\subsubsection{Compatibilité}

Un autre aspect important des caractéristiques d'une innovation (Tic) affectant son taux d'adoption est sa compatibilité perçue ou acceptabilité (Adegbidi, Mensah, Vidogbena, \& 
Agossou, 2012). La compatibilité est le degré avec lequel une innovation tic dans notre cas est perçue comme consistant avec les valeurs culturelles et de croyance des potentiels utilisateurs de l'innovation (Tic) (Ventkatesh et al., 2003). La Compatibilité aide les individus à donner un sens a une nouvelle innovation afin qu'elle soit regardée comme plus familier (Barakabitze et al., 2015). Dans notre cas, la manière de parler aux paysans à travers les centres d'appel téléphonique et même les agents par exemple de Ngasene dans les villages respectifs doit être conforme à certaines valeurs culturelles pour qu'ils (elles) adoptent/utilisent le nouveau système. Ce concept a été souligné par beaucoup d'études (tableau 2) et pays comme un facteur dans l'utilisation des Tics pour accéder et utiliser l'information de production agricole.

\subsubsection{Simplicité}

La Simplicité est le degré avec lequel une innovation (Tic) est perçue comme simple à comprendre et à utiliser. Toute nouvelle idée d'innovation doit être placé sous le continuum de complexite-simplicite. Certaines innovations sont claires, compréhensives aux yeux des utilisateurs potentiels alors que d'autres ne le sont pas (Carter \& Belanger, 2004). Dans beaucoup d'études, le mot Simplicité est utilisé en lieu et place de Complexité (Atkinson, 2007) étant donné qu'il affecte positivement l'utilisation de l'innovation (Tic). Au Kenya par exemple, les paysans trouvent que l'accès aux information agricoles de production à travers le téléphone portable est compliqué (pas simple) (KTM, 2013). La même observation a été faite par une étude en Tanzanie (Wulystan \& Andrew, 2013). Nous concluons alors que le concept Simplicité est un facteur dans l'utilisation des Tics.

\subsubsection{Observabilité}

L'Observabilité, aussi connue comme la Communibilité, démonstrabilité ou descriptabilite, est le degré par lequel les résultats d'une innovation (Tic) est visible par les autres (Adegbidi et al., 2012). Il est positivement relie a l'adoption (utilisation) des Tics sur l'agriculture de précision (Rezaei-Moghaddam \& Salehi, 2010). Dans un autre cas au Mali, les paysans utilisant le Tic Senekela affirment que les non-utilisateurs viennent à eux chaque mois pour des conseils agricoles (T. Palmer, 2014).

Nous pouvons conclure ici qu'il y'a bien une relation entre le concept d'Observabilité (devenant ainsi un facteur) et l'utilisation des Tics pour l'accès et l'utilisation de l'information agricole sur les Tics.

\subsubsection{Cout}

Le cout élevé des services Tics constitue un facteur limitant leur utilisation. Par exemple en Tanzanie, le coût des téléphones mobile combine et leur service, ont exclus beaucoup de pauvres ruraux paysans dans l'accès à TigoKilimo (Chung, 2015). Au Mali, 95\% des utilisateurs de SENEKELA trouvent que le coût du service est inabordable. De plus en Tanzanie, le cout était une barrière pour l'utilisation des Tics dans le domaine de l'information agricole sur les intrants. Nous concluons par conséquent, que le coût est un facteur dans l'utilisation de ces Tics dans les pays en voie de développement. 


\subsubsection{L'Influence Sociale}

Les paysans sont connus pour leur partage d'information entre eux. Par exemple, Edda Tandi Lwoga (2010) affirme que la majeur source d'information des paysans étaient principalement les locaux (voisins, amis et famille).

L'Influence Sociale est définie comme le degré avec lequel un individu est persuadé que les autres (importantes) personnes pensent qu'il doit utiliser le système (Ventkatesh et al., 2003). Il a été souligné que l'Influence Sociale a un effet significatif sur l'utilisation des Tics (Kaba, Diallo, \& Plaisent, 2006; Ventkatesh et al., 2003). Au Mali, les paysans (74\%) se partagent les informations agronomiques qu'ils reçoivent de SENEKELA(T. Palmer, 2015). Par conséquent, l'utilisation des Tics sur l'information agricole sur les intrants est affectée par le facteur Influence Sociale.

\subsubsection{La Qualité de l'Information délivrée}

L'information agricole sur les intrants doit être appropriée, précise et complète pour que le paysan l'utilise. Il a été observé au Kenya que parfois les paysans ont l'information agricole sur les intrants mais ne l'utilisent pas (KTM, 2013). Ils questionnent l'efficacité de cette information.

L'information relative à la disponibilité des intrants agricole et de leur prix a aussi été perçue comme 'inappropriée' par $72.5 \%$ des répondants dans le district de Gyandoot (Inde) (Meera, Jhamtani, \& Rao, 2004). Ce résultat était similaire à celui d'une autre étude conduit au Mali (T. Palmer, 2014), qui conclue que les paysans trouve l'information qui leur est délivrée incomplète. Au Kenya, il y'a toujours de la place pour une amélioration depuis qu'un large nombre de petits exploitants du pays (3,5 millions) travaille toujours sans intrants agricoles (KTM, 2013).

De plus, beaucoup de paysans n'ont pas d'information adéquate sur comment utiliser les intrants agricoles auxquels ils ont accès. En Ouganda, la valeur ajoutée d'une information dans le domaine agricole dépend de plusieurs caractéristiques : accessibilité, précision (justesse), pertinence et d'actualité (Kaddu, 2011). Pour qu'une information soit utilisable ou bénéfique, elle doit être opportune, compréhensible, provenant d'une source de confiance, inclusive et non subversive (Beardon, Munyampeta, Rout, \& Williams, 2005).

Par conséquent nous, concluons que l'utilisation (utilisation continuelle) des Tics pour l'accès et l'utilisation de l'information agricole sur les intrants est liée au facteur Qualité de l'Information délivrée.

\subsubsection{Savoir-faire Tics et Analphabétisme}

Pour accéder à l'information à travers les Tics, il est important de savoir comment faire et avoir le savoir-faire pour cela. Dutta et al. (2004) affirme que sans une population éduquée et consciente des Tics, aucune communauté ne peut complètement participer dans ce monde interconnecté. En plus, en Afrique sub saharienne, beaucoup de pays manque de programme d'alphabétisation et par conséquent, aucun résultat ne peut être durable (Kaddu, 2011). 
L'analphabétisme et un faible savoir-faire Tics sont des facteurs pour l'utilisation des Tics sur l'information agricole sur les intrants. Par exemple en Tanzanie, GSMA (2015) rapporte que l'analphabétisme et le faible niveau de technicité constitue des barrières majeures dans l'utilisation de Tigokilimo parce que l'utilisation de l'USSD (Unstructured Supplementary Service Data) nécessite que les utilisateurs naviguent à travers un menu textuel. Msoffe \& Ngulube (2016) affirment aussi que le faible niveau d'alphabétisation est une barrière à l'accès de l'information par les paysans.

Une autre étude menée en Inde sur l'utilisation des Tics dans le domaine agricole conduit pas Meera et al. (2004) trouve que l'éducation était positivement relie a la fréquente utilisation des services internet de Gyandoot et Warama.

En Serbie, Simin \& Janković (2014) affirme que l'adoption d'une innovation en agriculture est positivement liée au niveau d'éducation. Finalement, en Arabie Saoudite, AlGhaith et al. (2010) aussi affirment que l'utilisateur (adopteur) d'une nouvelle technologie nécessite un niveau d'éducation approprié. Par conséquent, nous concluons qu'il y a une relation entre le facteur alphabétisation, savoir-faire Tics et l'utilisation des Tics dans le domaine de l'information agricole (intrants).

\subsubsection{Conclusion}

Finalement, le codage libre dégage que l'avantage relatif, la simplicité, le compatibility, l'observabilité, l'influence sociale, le coût des Tics, le niveau d'alphabétisation, le savoir-faire Tics et la qualité de l'information délivrée affectent l'utilisation des Tics dans le cadre de l'accès à l'information agricole sur les intrants et l'utilisation de cette information (Table 2). 
Table 2. Concepts

\begin{tabular}{|c|c|c|}
\hline Concept & Auteurs (Evidence & Pays \\
\hline Avantage Relatif & $\begin{array}{l}\text { (Adegbidi et al., 2012; Al-Ghaith et al., 2010; } \\
\text { Atkinson, 2007; Carter \& Belanger, 2004; } \\
\text { Rezaei-Moghaddam \& Salehi, 2010; Rogers, } \\
\text { 1983; Surendran, 2012; Ventkatesh et al., 2003) }\end{array}$ & $\begin{array}{l}\text { Benin, Inde, } \\
\text { Indonésie, Iran, } \\
\text { Kenya, Mali, Nigeria, } \\
\text { Pakistan, Tanzanie, } \\
\text { Ouganda, Sénégal }\end{array}$ \\
\hline Compatibilité & $\begin{array}{l}\text { (Adegbidi et al., 2012; Atkinson, 2007; Carter } \\
\& \text { Belanger, 2004; T. Palmer, 2015; Rezaei- } \\
\text { Moghaddam \& Salehi, 2010; Rogers, 1983; } \\
\text { Surendran, 2012) }\end{array}$ & $\begin{array}{l}\text { Benin, Inde, } \\
\text { Indonésie, Iran, } \\
\text { Kenya, Mali, Nigeria, } \\
\text { Pakistan, Tanzanie, } \\
\text { Ouganda, Sénégal }\end{array}$ \\
\hline Complexité/Simplicité & $\begin{array}{l}\text { (Adegbidi et al., 2012; Al-Ghaith et al., 2010; } \\
\text { Atkinson, 2007; Carter \& Belanger, 2004; } \\
\text { Dandedjrohoun, Diagne, \& Biaou, 2012; } \\
\text { Rezaei-Moghaddam \& Salehi, 2010; Rogers, } \\
\text { 1983; Surendran, 2012; Ventkatesh et al., 2003) }\end{array}$ & $\begin{array}{l}\text { Benin, Inde, } \\
\text { Indonésie, Iran, } \\
\text { Kenya, Mali, Nigeria, } \\
\text { Pakistan, Tanzanie, } \\
\text { Ouganda, Sénégal }\end{array}$ \\
\hline
\end{tabular}

(Adegbidi et al., 2012; Atkinson, 2007; Carter

Observabilité \& Belanger, 2004; Rezaei-Moghaddam \&

Benin, Iran, Mali, Salehi, 2010; Rogers, 1983; Singh et al., 2016; Tanzanie, Uganda Surendran, 2012; Ventkatesh et al., 2003)

(Atajeromavwo, Ekruyota, Eti, \& Oyefia, 2010 ;

Qualité de l'Information Hatakka \& De, 2011; Heeks \& Molla, 2009; Mittal \& Mehar, 2012; Msoffe \& Ngulube, 2016; Myagro, n.d.; T. Palmer, 2014, 2015)
Inde, Kenya, Mali, Nigeria, Tanzanie, Pakistan, Ouganda

\section{Cout}

$$
\begin{array}{ll} 
& \text { (GSMA, 2015; Kaba et al., 2006; E T Lwoga, } \\
\text { Influence Sociale } & \text { Stilwell, \& Ngulube, 2011; Edda Tandi Lwoga, } \\
\text { 2010; N. Palmer, 2011; Ventkatesh et al., 2003; } & \text { Wulystan \& Andrew, 2013) }
\end{array}
$$$$
\text { Savoir-faire Tics }
$$

(Palmer, 2014; GSMA, 2015; Siraj, 2010; Adegbidi et al., 2012)
Mali, Tanzanie, Uganda
Guinée Conakry, Iran, Mali, Tanzanie, Ouganda
(Kaddu, 2011; Palmer, 2014; Chung, 2015; Kameswari et al., 2011; Atajeromavwo et al., 2010;)
Ouganda, Mali, Tanzanie, Inde

(Adegbidi et al., 2012; Chung, 2015;

Alphabétisation Glendenning \& Ficarelli, 2012; T. Palmer, 2014; Siraj, 2010)

Benin, Tanzanie, Mali, Pakistan

Source: adaptée de Kante et al. (2016) 


\subsubsection{Codage Axial}

Après avoir terminé le codage libre, nous avons regroupé les différents concepts en catégorie. Les différentes catégories qui ont émergé sont: la perception, l'influence des paysans les uns sur les autres, le coût des Tics, la qualité de l'information délivrée, l'alphabétisation et le savoir-faire Tics.

La perception des paysans (avantage relatif, simplicité, compatibilité) sur les Tics à un effet sur leurs utilisations de ces Tics. L'avantage relatif est une perception et dépend de tout un chacun. La simplicité aussi est une perception comme suggère dans le domaine de la production agricole par Adegbidi et al. (2012), dans le domaine des Tics et la santé par AmiNarh \& Williams (2012) et dans le domaine de l'éducation par Atkinson (2007). La compatibilité a aussi été définie par plusieurs études comme une perception et dans notre cas, nous l'avons rangé dans cette catégorie.

Par contre, la catégorie de l'observabilité est sujette à discussion. Ce mot a été défini comme une perception par beaucoup d'autres (Atkinson, 2007; Dandedjrohoun et al., 2012; Ventkatesh et al., 2003). Mais le concept Observabilité, selon Rezaei-Moghaddam \& Salehi (2010) est très complique. Ils le divisèrent alors en deux concepts à savoir Visibilité et Démonstrabilité des Résultats. La démonstrabilité des résultats fait allusion à «la tangibilité des résultats en utilisant une innovation » et la visibilité fait allusion " dans quelle mesure les résultats sont visible à un potentiel utilisateur ». En appliquant ceux-ci à notre contexte, «la tangibilité des résultats en utilisant une innovation » serait « la tangibilité d'une amélioration positive de la connaissance du paysans sur les intrants agricoles à travers l'utilisation des Tics » et « dans quelle mesure les résultats sont visible a un potentiel utilisateur » serait « la visibilité de la connaissance accrue d'un paysan utilisant ces Tics par un autre paysan grâce à une interaction entre les paysans ». En conclusion, le concept d'Observabilité (dans notre cas) est une perception mais obtenue grâce à un échange entre les paysans. Nous l'incluons alors dans la catégorie de l'influence que les paysans ont les uns sur les autres dans l'utilisation des Tics pour avoir l'information agricole sur les intrants et utiliser cette information. L'influence Sociale aussi est un élément de cette catégorie.

Le coût excessif, l'alphabétisation, le savoir-faire Tics et la qualité de l'information délivrée ne pouvaient pas être inclus dans une autre catégorie. Par conséquent ces quatre concepts deviennent des catégories. Le Tableau 3 donne une vue d'ensemble des résultats du codage axial.

\subsubsection{Codage sélectif}

Le codage sélectif consistait à identifier les propriétés de ces différentes catégories que nous avions extraites. En d'autres termes, il s'agissait de savoir si elles affectaient de façon positive ou négative l'utilisation des Tics.

La perception a été identifiée comme ayant un effet positif sur l'utilisation des Tics dans les études que nous avions sélectionnés. Par exemple au Benin, il a été trouvé que l'utilisation des Tics nécessite des attitudes positives (Adegbidi et al., 2012).

Les paysans ont une influence positive les uns sur les autres dans l'utilisation des Tics. Ceci a été note au Mali par T. Palmer (2015), en Zambie par Bwalya (2009), au Pakistan par 
Siraj (2010), en Inde par Kameswari et al. (2011) et en Ouganda (Kaddu, 2011).

La Qualité de l'Information délivrée à un effet positif sur l'utilisation des Tics par les paysans. Par exemple en Arabie Saoudite, Al-Ghaith et al. (2010) étiqueta la Qualité de l'information comme Qualité du e-service et trouve qu'elle affecte positivement l'utilisation des Tics. Un résultat similaire a été observé en Tanzanie par Msoffe \& Ngulube (2016). Donc son effet est positif.

Une barrière dans l'utilisation des Tics est le coût élevé des Tics (et services). Ceci a été noté par plusieurs études dans plusieurs pays en voie de développement comme la Tanzanie, le Mali, le Pakistan, l'Inde, la Zambie, le Kenya et l'Ouganda(Chung, 2015; Kaddu, 2011; Kalusopa, 2005; Kameswari et al., 2011; KTM, 2013; T. Palmer, 2014; Siraj, 2010).

L'alphabétisation et le savoir-faire Tics sont des facteurs affectant positivement l'utilisation des Tics. Ceux-ci a été démontré par plusieurs études (Al-Ghaith et al., 2010; Simin \& Janković, 2014).

Dues à ces facteurs, les Tics font face à un problème de faible utilisation. Par exemple au Mali Senekela a seulement 177.817 utilisateurs (GSMA, 2015). Ce nombre est très petit compare aux potentiels utilisateurs dans un pays ou $73 \%$ de la population évolue dans le secteur agricole. De plus l'opérateur Orange a une part de marche de $2 / 3$ des $12.832 .814^{2}$ utilisateurs de téléphones portables dans le pays. Un autre Tic (MyAgro) a une clientèle de seulement 3500 paysans au Mali et au Sénégal (Myagro, n.d.). Ce nombre limite d'utilisateur au mali et au Sénégal était comparable aux utilisateurs de Tigokilimo en Tanzanie, ou ce service aussi avait seulement atteint $6 \%$ des potentiels utilisateurs sur le marché (Chung, 2015). Les catégories et leur propriété respective sont détaillées dans la Table 3.

Table 3. Catégories

\begin{tabular}{lll}
\hline Catégorie & Concepts & Propriétés \\
\hline Perception & Avantage Relatif, Compatibilité et Simplicité, & Effet Positif \\
Influence des uns sur les autres & Observabilité et l'Influence Sociale & Effet Positif \\
\hline Qualité de l'Information & Complet, pertinence, actualité et approprie & Effet Positif \\
Coût & Cout élevé & Effet Négatif \\
Alphabétisation & Alphabétisation, savoir écrire et lire & Effet Positif \\
\hline Savoir-faire Tics & Technicité, Savoir-faire Tics & Effet Positif \\
\hline
\end{tabular}

2 Autorité Malienne de Régulations des Télécommunications 2015 


\section{DISCUSSION}

Les pays en voie de développement ont implémenté des services Tics pour diffuser de l'information sur les intrants agricoles. L'objectif principal des Tics est de diffuser ces informations. La meilleure façon de diffuser de l'information agricole (Bosch et al., 2012) sur les intrants n'est pas une tâche facile surtout au niveau des petits producteurs des pays en voie de développement. Nous avons fait ressortir les facteurs qui affectent l'utilisation de ces Tics dans les pays en voie de développement et la façon dont ils affectent ces Tics.

Nos résultats sont similaires à ceux de deux autres études menées dans les pays en voie de développement à savoir Benin et Iran dans le même contexte d'adoption des Tics (Adegbidi et al., 2012; Rezaei-Moghaddam \& Salehi, 2010). Cependant, ces études n'avaient pas fait ressortir les facteurs Qualité de l'Information, l'Influence Sociale et le Coût comme ayant un effet sur l'utilisation des Tics. De plus nos facteurs sont similaires à ceux de la Diffusion de l'Innovation (Diffusion of innovation Theory - DOI/IDT). Cette théorie tente d'expliquer l'adoption d'une innovation (Tic) par les utilisateurs potentiels (Atkinson, 2007). Cela confère à nos résultats une base théorique.

Certains de nos facteurs ont été identifiés dans les pays développés comme ayant un effet sur l'utilisation des Tics. Par exemple, l'avantage relatif, la complexité et la compatibilité ont été identifiées des facteurs ayant un effet négatif sur l'utilisation des ERP(Enterprise Resource Planning) adoption en Hollande dans le domaine de l'horticulture (Verdouw, Robbemond, \& Wolfert, 2015).

\section{CONCLUSION}

Notre revue et analyse des facteurs affectant l'utilisation des Tics dans le domaine de l'information sur les intrants agricoles par les paysans dans les pays en voie de développement ont montré qu'en dépit de la disponibilité de ces Tics, les paysans (petits producteurs) ont toujours des difficultés à accéder à l'information sur les intrants agricole et utiliser cette information. Ceci a conduit à une contribution insignifiante des Tics dans le domaine agricole. Nous avons identifié les facteurs avantage relatif, compatibilité, simplicité, observabilité, influence sociale, l'alphabétisation, le savoir-faire Tics et la qualité de l'information comme ayant un effet positif sur l'utilisation des Tics dans l'accès à l'information sur les intrants agricole et l'utilisation de cette information tandis que le coût a un effet négatif sur ces Tics dans la même logique. Cette revisite de l'article (Kante et al., 2016) était nécessaire car elle a permis d'actualiser la revue littéraire et de plus a aussi identifié deux importants facteurs qui sont l'alphabétisation et le savoir-faire Tics ainsi que leurs propriétés.

Les résultats de cette étude suggèrent des investigations futures à mener sur l'utilisation des Tics par les petits producteurs pouvant améliorer leurs conditions de vie. Par exemple, une étude pourra être menée dans le même domaine de la production agricole (information sur les intrants) pour confirmer ou informer nos hypothèses. De plus, d'autres facteurs pourraient être identifies. 


\section{REMERCIEMENTS}

This material is based upon work supported by the United States Agency for International Development, as part of the Feed the Future initiative, under the CGIAR Fund, award number BFS-G-11-00002, and the predecessor fund the Food Security and Crisis Mitigation II grant, award number EEM-G-00-04-00013.

\section{BIBLIOGRAPHIE}

Adegbidi, A., Mensah, R., Vidogbena, F., \& Agossou, D. (2012). Determinants of ICT use by rice farmers in Benin : from the perception of ICT characteristics to the adoption of the technology. Journal of Research in International Business and Management, 2(11), 273-284.

AGRA. (2014). Improving fertilizer supplies for African farmers. Nairobi. Retrieved from http://iirr.org/wp-content/uploads/2015/09/Fertilizer-supply_print-1.pdf

Aker, J. C. (2011). Dial “ A ” for Agriculture : Using Information and Communication Technologies for Agricultural Extension in Developing Countries. Agricultural Economics, 42(6), 631-647. http://doi.org/10.1111/j.1574-0862.2011.00545.x

Al-Ghaith, W., Sanzogni, L., \& Sandhu, K. (2010). Factors Influencing the Adoption and Usage of Online Services in Saudi Arabia. Electronic Journal of Information Systems in Developing Countries, 40(1), 1-32.

Ami-Narh, J. T., \& Williams, P. a H. (2012). A Revised UTAUT Model to Investigate E-health Acceptance of Health Professionals in Africa. Journal of Emerging Trends in Computing and Information Sciences, 3(10), 1383-1391.

Angelucci, F., Gourichon, H., Mas Aparisi, A., \& Witwer, M. (2013). Monitoring and analysing food and agricultural policies in Africa MAFAP Synthesis Report Series.

Atajeromavwo, E. J., Ekruyota, G., Eti, I., \& Oyefia, E. (2010). An Evaluation of the Relevance of Information and Communication Technologies (ICT) to Agricultural and Rural Development in Niger Delta of Nigeria. African Research Review, 4(1), 401-414.

Atkinson, N. L. (2007). Developing a questionnaire to measure perceived attributes of eHealth innovations. American Journal of Health Behavior, 31, 612-621.

http://doi.org/10.5993/AJHB.31.6.6

Barakabitze, A. A., Kitindi, J. E., Sanga, C., Shabani, A., Philipo, J., \& Kibirige, G. (2015). NEW TECHNOLOGIES FOR DISSEMINATING AND COMMUNICATING AGRICULTURE KNOWLEDGE AND INFORMATION: CHALLENGES FOR AGRICULTURAL RESEARCH INSTITUTES IN TANZANIA. The Electronic Journal of Information Systems in Developing Countries, 70, 1-22.

Beardon, H., Munyampeta, F., Rout, S., \& Williams, G. M. (2005). ICT for development : empowerment or exploitation? Learning from the Reflect ICTs project. Human Development. London. Retrieved from reflect-action.org

Bosch, M., Rodrigues, M., Hopkins, R., Mamani, V., Chavarria, H., Espinel, B., \& Brossard, F. (2012). Agriculture and ICT. eLac, 18, 1-12.

Bwalya, K. J. (2009). FACTORS AFFECTING ADOPTION OF E-GOVERNMENT IN ZAMBIA. Electronic Journal of Information Systems in Developing Countries, 38(4), 1-13.

Carter, L., \& Belanger, F. (2004). Citizen adoption of electronic government initiatives. 37th Annual Hawaii International Conference on System Sciences, 2004. Proceedings of the, O(C), $10 \mathrm{pp}$. http://doi.org/10.1109/HICSS.2004.1265306 
Charmaz, K. (2006). Constructing Grounded Theory: A Practical Guide Through Qualitative Analysis. (D. Silverman, M. Bloor, N. Denzin, B. Glassner, J. Gubrium, A. Murcott, \& J. Potter, Eds.) (First). London: Sage Publications.

Choupal, O. (2011). About e-Choupal. Retrieved from http://echoupal.com/DefaultTop.aspx\#

Chung, Y. S. Case study: Tigo Kilimo, Tanzania. (PUBmed, Ed.), 24GSMA 48-51 (March 15, 2015). London. http://doi.org/10.1016/S1360-8592(98)80013-2

CICC. (2003). Success and Future Prospects of "Initiative for Multimedia Information Technology Toward 21 (pp. 1-6).

Dandedjrohoun, L., Diagne, a, \& Biaou, G. (2012). Determinants of diffusion and adoption of improved technology for rice parboiling in Benin. Review of Agricultural and Environmental Studies, 93(2), 171-191. Retrieved from http://www.raestud.eu/pdf/171-191-ncho.pdf

Dangi, N., \& Singh, H. (2010). e-Choupal : Hope or Hype ? Neeraj Dangi and Harjit Singh Department of Management, Institute of Management Education, Sahibabad ( Ghaziabad ), India. American Journal of Economics and Business Administration, 2(2), 179-184.

Glendenning, C. J., \& Ficarelli, P. P. (2012). The Relevance of Content in ICT Initiatives in Indian Agriculture. International Food Policy Research Institute.

GSMA. Case study: Orange Senekela, Mali (2015). http://doi.org/10.1016/S1360-8592(98)80013-2

Harrod, J., \& Jamsen, P. (2010). Farmer Organizations Work Better with ICT. In ICT in Agriculutre: Connecting Smallholders to Knowledge, Networks, and Institutions (pp. 173-202).

Hatakka, M., \& De, R. (2011). Development, capabilities and technology: an evaluative framework. ... Developing Countries: Partners for Development ..., (May 2011). Retrieved from http://www.diva-portal.org/smash/record.jsf?pid=diva2:486303

Heeks, R., \& Molla, A. (2009). Impact Assessment of ICT-for-Development Projects: A Compendium of Approaches. Development Informatics Working Paper No 36. Retrieved from http://www.sed.manchester.ac.uk/idpm/research/publications/wp/di/index.htm

Kaba, B., Diallo, A., \& Plaisent, M. (2006). Explaining the factors influencing cellular phones use in Guinea. The Electronic Journal of Information Systems in Developing Countries, 28(3), 1-7. Retrieved from http://www.ejisdc.org/ojs2../index.php/ejisdc/article/view/341

Kaddu, S. B. (2011). INFORMATION AND COMMUNICATION TECHNOLOGIES' (ICTS) CONTRIBUTION TO THE ACCESS AND UTILISATION OF AGRICULTURAL INFORMATION BY THE RURAL WOMEN IN UGANDA. Makerere University.

Kalusopa, T. (2005). The challenges of utilizing information communication technologies (ICTs) for the small-scale farmers in Zambia. Library Hi Tech, 23(3), 414-424. http://doi.org/10.1108/07378830510621810

Kameswari, V. L. V., Kishore, D., \& Gupta, V. (2011). ICTs for agricultural extension: a study in the Indian Himalayan region. Electronic Journal of Information Systems in Developing Countries, $48(3), 1-12$.

Kante, M., Oboko, R., \& Chepken, C. (2016). Factors affecting the use of ICTs on agricultural input information by farmers in developing countries. AIMS Agriculture and Food, 1(3), 315-329. http://doi.org/10.3934/agrfood.2016.3.315

KTM. (2013). Kenya Markets Trust - Agricultural Inputs. Retrieved January 18, 2016, from http://www.kenyamarkets.org/what-we-do/the-market-assistance-programme/agricultural-inputs 
Lwoga, E. T. (2010). BRIDGING THE AGRICULTURAL KNOWLEDGE AND INFORMATION DIVIDE: THE CASE OF SELECTED TELECENTERS AND RURAL RADIO IN TANZANIA. Electronic Journal of Information Systems in Developing Countries, 43(6), 1-14.

Lwoga, E. T., Stilwell, C., \& Ngulube, P. (2011). Access and use of agricultural information and knowledge in Tanzania. Library Review, 60(5), 383-395. http://doi.org/10.1108/00242531111135263

Meera, S. N., Jhamtani, A., \& Rao, D. U. M. (2004). INFORMATION AND COMMUNICATION TECHNOLOGY IN AGRICULTURAL DEVELOPMENT : A COMPARATIVE ANALYSIS OF THREE PROJECTS FROM INDIA Shaik . N . Meera, Anita Jhamtani, and D . U . M . Rao. Agricultural Research \& Extension Network, (135), 2-15.

MFARMERKENYA. (n.d.). MFARMER. Retrieved April 25, 2016, from http://www.mfarmerkenya.org/

Mittal, S., \& Mehar, M. (2012). How Mobile Phones Contribute to Growth of Small Farmers? Evidence from India. Quarterly Journal of International Agriculture, 51(3), 227-244.

Msoffe, G. E. P., \& Ngulube, P. (2016). Agricultural Information Dissemination in Rural Areas of Developing Countries : A Proposed Model for Tanzania. Electronic Journal of Information Systems in Developing Countries, 26(2), 169-187.

Murage, A., Midega, C., Pittchar, J., \& Khan, Z. (2013). Potential uptake determinants of climatesmart push-pull technology in drier agro-ecological zones of eastern Africa. In the 4th International Conference of the African Association of Agricultural Economists, (p. 24). Retrieved from http://ageconsearch.umn.edu/bitstream/161511/2/Murage A.W., Midega C.A.O, Pittchar J.O., Khan Z.R.pdf

Myagro. (n.d.). myAGRO. Retrieved December 2, 2015, from http://www.myagro.org/meet-ourfarmers/

Odhiambo, F. (2014). Unlocking the market. In CTA (Ed.), Revolutionising finance for agri-value chains. Nairobi: ICT4AG. Retrieved from http://ictupdate.cta.int/Feature-Articles/Unlocking-themarket

Orange. (2014). services for agriculture in Africa. Retrieved from http:///www.orange.com/.../Catalogue_Orange_mAgri_JAN14_external

Ouedrago, J. E. evaluation des besoins en informations agricoles dans les etats du groupe afriquecaraibes-pacifique (acp), 53Centre Technique de cooperation Agricole et rurale (CTA) 1-163 (2008). http://doi.org/10.1017/CBO9781107415324.004

Palmer, N. (2011). Utiliser les TIC pour permettre aux petits exploitants d'accéder aux systèmes d'innovation agricole (e-sourcebook).

Palmer, T. (2014). mAgri Rapport initial sur Orange. Retrieved from http://www.gsma.com/mobilefordevelopment/programme/magri/early-results-from-orangesenekela-data-and-insights-from-the-baseline-version-francaise-disponible

Palmer, T. (2015). Orange Sènèkèla Midline. Retrieved from http://www.gsma.com/mobilefordevelopment/wpcontent/uploads/2015/09/GSMA_Senekela_Midline.pdf

Pshenichnaya, N., \& Clause, V. mAgri Case Study: Nokia Life Agriculture Service (2013). Retrieved from http://www.gsma.com/mobilefordevelopment/wpcontent/uploads/2013/03/GSMAmAgri_Nokia_Case_Study.pdf

Rezaei-Moghaddam, K., \& Salehi, S. (2010). Agricultural specialists' intention toward precision agriculture technologies: Integrating innovation characteristics to technology acceptance model. 
African Journal of Agricultural Research, 5(March), 1191-1199.

http://doi.org/10.5897/AJAR09.506

Rogers, E. M. (1983). DIFFUSION OF Third Edition (Third Edit). New York: Macmillan Publishing. http://doi.org/82-70998

Simin, M., \& Janković, D. (2014). Applicability of Diffusion of Innovation Theory in Organic Agriculture. Economics of Agriculture, 2014(61), 517-531. http://doi.org/10.5937/ekoPolj1402517T

Singh, M., Bhanotra, A., L, N., Wani, S. A., \& Kumar, M. (2016). Mobile Phone Technology-An Eminent ICT Tool for Better Family Farming Mobile Phone Technology- An Eminent ICT Tool for Better Family Farming. In Family Farming and Rural development (pp. 287-291).

Siraj, M. (2010). A model for ICT based services for agriculture extension in Pakistan. Retrieved from http://r4d.dfid.gov.uk/PDF/Outputs/Misc_Infocomm/60818-extensionmodel-Pakistan.pdforg

Siyao, P. O. (2012). Barriers in accessing Agricultural Information in Tanzania With a Gender Perspective : the Case study of small-scale sugar Cane growers in Kilombero District. Electronic Journal of Information Systems in Developing Countries, 1-19.

Sukhpal, S. (Indian I. of. (2004). Leveraging ICT for Agricultural Development: A case study of eChoupals of ITC Sukhpal Singh*, VIII(1), 1-6.

Surendran, P. (2012). Technology Acceptance Model : A Survey of Literature. International Journal of Business and Social Research, 2(4), 175-178.

Traoré, A., Mamy, S., JBélières, J.-F., \& Hilhorst, T. (2011). Quelles évolutions des systèmes de production céréaliers au Mali ?, 54-56.

Ueno, T., \& Yoshida, K. (2012). Nikkei BP Consulting, Inc. | Newsrelease. Retrieved February 1, 2016, from http://consult.nikkeibp.co.jp/english/news/120420/

Urquhart, C., \& Fernández, W. (2013). Using grounded theory method in information systems: the researcher as blank slate and other myths. Journal of Information Technology, 28(3), 224-236. http://doi.org/10.1057/jit.2012.34

Ventkatesh, V., Morris, M. G., Hall, M., Davis, G. B., Davis, F. D., \& Walton, S. M. (2003). USER A CCEPTANCE OF I NFORMATION T ECHNOLOGY : TOWARD A UNIFIED VIEW. MIS Quarterly, 27(3), 425-478.

Verdouw, C. N., Robbemond, R. M., \& Wolfert, J. (2015). ERP in agriculture: Lessons learned from the Dutch horticulture. Computers and Electronics in Agriculture, 114, 125-133. http://doi.org/10.1016/j.compag.2015.04.002

Wolfswinkel, J. F., Furtmueller, E., \& Wilderom, C. P. M. (2011). Using grounded theory as a method for rigorously reviewing literature. European Journal of Information Systems, 22(1), 45-55. http://doi.org/10.1057/ejis.2011.51

World Bank. (2011). ICT in agriculture: connecting amallholders to knowledge, networks, and institutions. World, (64605), 424. http://doi.org/64605

Wulystan, P. M., \& Andrew, C. M. (2013). Using Information and Communication Technologies for Enhancing the Accessibility of Agricultural Information for Improved Agricultural Production in Tanzania. Electronic Journal of Information Systems in Developing Countries, 56(1), 1-14.

ZANTEL. (2013). Zantel launches new mobile service for farmers. Retrieved February 1, 2016, from http://www.zantel.com/press_release/Zantel-M-AGRICULTURE-release.pdf 